\title{
Astrocytes are central in the pathomechanisms of vanishing white matter
}

\author{
Stephanie Dooves, ${ }^{1}$ Marianna Bugiani, ${ }^{1,2}$ Nienke L. Postma, ${ }^{1}$ Emiel Polder, ${ }^{1}$ Niels Land, ${ }^{1}$ Stephen T. Horan, ${ }^{1}$ \\ Anne-Lieke F. van Deijk, ${ }^{3}$ Aleid van de Kreeke, ${ }^{1}$ Gerbren Jacobs, ${ }^{1}$ Caroline Vuong, ${ }^{1}$ Jan Klooster, ${ }^{4}$ Maarten Kamermans, ${ }^{4}$ \\ Joke Wortel, ${ }^{5}$ Maarten Loos, ${ }^{6}$ Lisanne E. Wisse, ${ }^{1}$ Gert C. Scheper, ${ }^{1}$ Truus E.M. Abbink, ${ }^{1}$ Vivi M. Heine, ${ }^{1,7}$ and Marjo S. van der Knaap ${ }^{1,5}$ \\ 'Department of Pediatrics/Child Neurology, and ${ }^{2}$ Department of Pathology, VU University Medical Center, Amsterdam, Netherlands. ${ }^{3}$ Department of Molecular and Cellular Neurobiology, \\ Center for Neurogenomics and Cognitive Research, Neuroscience Campus Amsterdam, VU University, Amsterdam, Netherlands. ${ }^{\circ}$ Department of Retinal Signal Processing, \\ Netherlands Institute for Neuroscience - KNAW, Amsterdam, Netherlands. ${ }^{5}$ Department of Functional Cenomics, Center for Neurogenomics and Cognitive Research, Neuroscience Campus Amsterdam, \\ VU University, Amsterdam, Netherlands. ' ${ }^{6}$ Sylics (Synaptologics BV), Amsterdam, Netherlands. 'Department of Complex Trait Cenetics, Center for Neurogenomics and Cognitive Research, \\ Neuroscience Campus Amsterdam, VU University, Amsterdam, Netherlands.
}

\begin{abstract}
Vanishing white matter (VWM) is a fatal leukodystrophy that is caused by mutations in genes encoding subunits of eukaryotic translation initiation factor 2B (elF2B). Disease onset and severity are codetermined by genotype. White matter astrocytes and oligodendrocytes are almost exclusively affected; however, the mechanisms of VWM development remain unclear. Here, we used VWM mouse models, patients' tissue, and cell cultures to investigate whether astrocytes or oligodendrocytes are the primary affected cell type. We generated 2 mouse models with mutations (Eif2b5 Arg191His/Arg191His and Eif2 b4 Arg484Trp/Arg484Trp) that cause severe VWM in humans and then crossed these strains to develop mice with various mutation combinations. Phenotypic severity was highly variable and dependent on genotype, reproducing the clinical spectrum of human VWM. In all mutant strains, impaired maturation of white matter astrocytes preceded onset and paralleled disease severity and progression. Bergmann glia and retinal Müller cells, nonforebrain astrocytes that have not been associated with VWM, were also affected, and involvement of these cells was confirmed in VWM patients. In coculture, VWM astrocytes secreted factors that inhibited oligodendrocyte maturation, whereas WT astrocytes allowed normal maturation of VWM oligodendrocytes. These studies demonstrate that astrocytes are central in VWM pathomechanisms and constitute potential therapeutic targets. Importantly, astrocytes should also be considered in the pathophysiology of other white matter disorders.
\end{abstract}

\section{Introduction}

Vanishing white matter (VWM) (OMIM 603896), one of the most prevalent genetically determined brain white matter disorders (leukodystrophies) in children (1), is clinically characterized by progressive motor dysfunction, mainly cerebellar ataxia, and less prominent cognitive decline. Occasional epileptic seizures may occur, but severe epilepsy is not a feature. Brain MRI shows a diffuse leukoencephalopathy with progressive white matter rarefaction and cystic degeneration (1). VWM is caused by mutations in the genes EIF2B1-EIF2B5, encoding subunits $\alpha-\varepsilon$ of eukaryotic translation initiation factor 2B (eIF2B) $(2,3)$. Although eIF2B has a housekeeping function and is ubiquitously expressed, VWM almost exclusively affects the brain white matter. Investigations into how eIF2B gene mutations specifically cause a brain disorder and the development of treatments for VWM are hampered by a lack of representative mouse models of the disease.

VWM may present at any age between birth and senescence $(1,4)$. Age of onset and clinical severity are inversely related and influenced by the genotype $(5,6)$. Antenatal-onset VWM presents

Authorship note: S. Dooves and M. Bugiani contributed equally to this work. V.M. Heine and M.S. van der Knaap are co-last authors.

Conflict of interest: The authors have declared that no conflict of interest exists. Submitted: July 27, 2015; Accepted: January 28, 2016.

Reference information: J Clin Invest. 2016;126(4):1512-1524. doi:10.1172/JCI83908. with intrauterine growth restriction and, at birth, severe encephalopathy, cataracts, and internal organ abnormalities; death occurs within a few months (7). The most common form has its onset between 2 and 6 years of age; death occurs within a few years (1). Adult-onset VWM is characterized by slowly progressive encephalopathy and death after several decades (4). Apart from ovarian failure, extracerebral organs are not affected in patients with onset after infancy. The disease is incurable (1).

eIF2B is essential for initiating the translation of mRNAs into peptides and regulates the translation rate in diverse conditions (8). It catalyzes the guanine nucleotide exchange on eIF2, converting inactive eIF2-GDP into active eIF2-GTP. During each round of translation initiation, eIF2.GTP brings initiator methionyl-transfer RNA (methionyl-tRNA) to the ribosome to recognize the start codon. eIF2B is therefore referred to as a guanine nucleotide exchange factor (GEF). Most, but not all, eIF2B mutations decrease its GEF activity in vitro (9-11). No correlation has been found between the degree of in vitro reduction of eIF2B GEF activity and clinical severity $(9,12)$, and no evidence has been provided that eIF2B mutations decrease protein synthesis or compromise cell viability in patients' skin fibroblasts and lymphoblasts (13-15). No clear clue for VWM pathophysiology has been obtained from these biochemical and molecular studies.

The neuropathology of human VWM brains at end-stage disease shows a sparing of brain gray matter structures, contrasting 
A

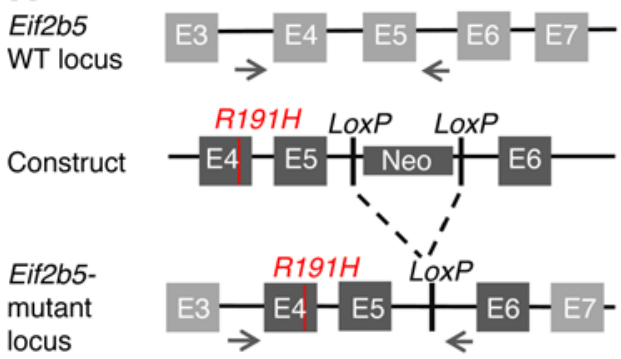

B

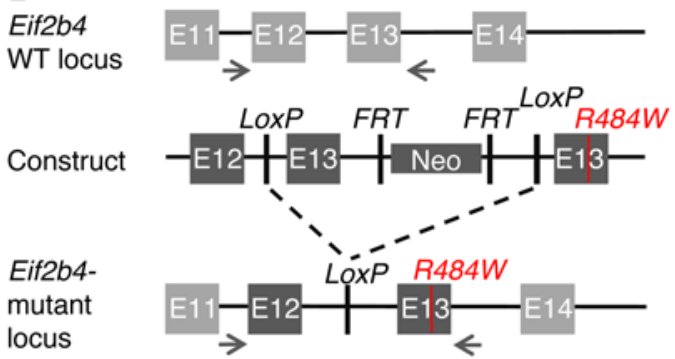

C
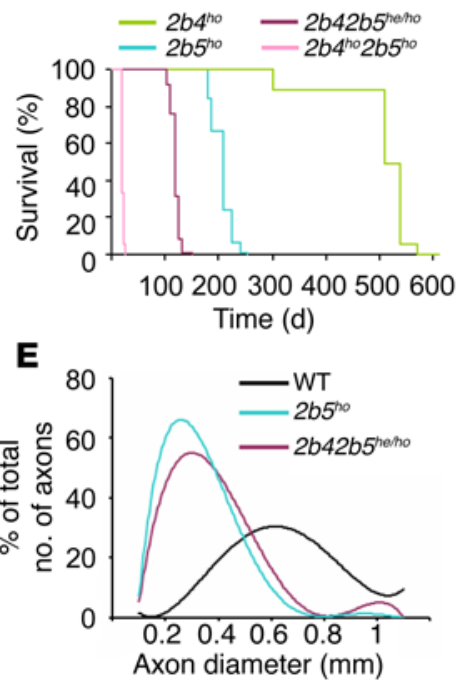

D
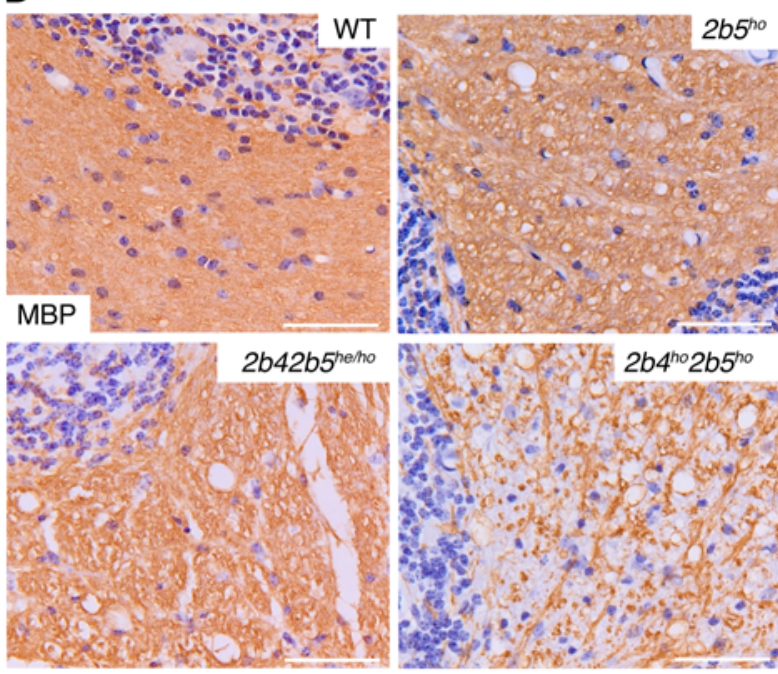

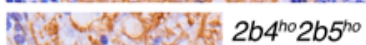

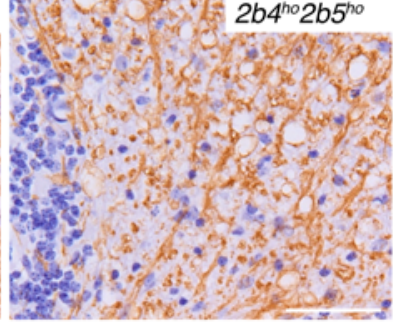

Figure 1. Generation of VWM mouse models. (A) $2 b 5^{\text {ho }}$ mice were generated by introducing a construct into the Eif $2 b 5$ gene locus consisting of exons $4-6$, including a point mutation in exon 4 . (B) $2 b 4^{\text {ho }}$ mice were generated by introducing a construct into the Eif $2 b 4$ gene locus consisting of exons 12 and 13 , including a point mutation in exon 13. (C) Kaplan-Meier graph shows the reduced lifespan of VWM mutant mice, with an average survival of 19 months for $2 b 4^{\text {ho }}$ mice $(n=9) ; 8$ months for $2 b 5^{\text {ho }}$ mice $(n=19) ; 4$ months for $2 b 42 b 5^{\text {he/ho }}$ mice $(n=12)$; and 3 weeks for $2 b 4^{\text {ho }} 2 b 5^{\text {ho }}$ mice $(n=6)$. (D) Staining for MBP showed vacuolization of the cerebellar white matter in 7-month-old $2 b 5^{\text {ho }}$, 4-month-old $2 b 42 b 5^{\text {he/ho }}$, and P21 $2 b 4^{\text {ho }} 2 b 5^{\text {ho }}$ mice, but not in 7-month-old WT mice. Scale bars: $50 \mu \mathrm{m}$. (E) The distribution of axonal diameters was skewed to the smaller diameters in $2 b 5^{\text {ho }}$ and $2 b 42 b 5^{\text {he/ho }}$ mice $(n=92 \mathrm{WT}$ mice; $n=1002 b 5^{\text {ho }}$ mice; $n=912 b 42 b 5^{\text {he/ho }}$ mice). Immunostainings are representative images from at least 3 experiments.

with severe white matter abnormalities, including a diffuse lack of myelin and cystic degeneration $(1,16)$. Reactive astrogliosis is disproportionately meager, considering the degree of tissue damage. Astrocytes are dysmorphic (17-20), immature (20,21), and overexpress the $\delta$-isoform of GFAP (20). Lack of myelin coexists with strikingly increased numbers of premyelinating oligodendrocyte progenitor cells (OPCs) $(20,21)$. High-molecular-weight (HMW) hyaluronan, an extracellular matrix component produced by astrocytes and known to inhibit OPC maturation (22), accumulates in VWM patients' white matter (21). The degree of white matter damage, the amounts of HMW hyaluronan locally present, and the increase in OPC numbers co-vary (21).

Although previous studies $(18,20,21)$ have suggested a role for astrocytes in the pathophysiology of VWM, this has not been confirmed. To explore the cellular pathophysiology of VWM and its dynamics throughout the disease course, and in view of future treatment strategies, we generated 2 new mutant mouse strains with a homozygous mutation in Eif 264 or Eif $2 b 5$ and crossed them with double mutants. We confirmed the relevance of the mutant mice for studies of the human disease by showing that they recapitulate the clinical and neuropathologic features as well as the variation in disease severity seen in human VWM. Patients' material confirmed novel findings in the mutant mice. We used cocultures of astrocytes and OPCs from mutant and WT mice to identify the primarily affected cell type in VWM.

\section{Results}

VWM mouse models. Two single mutants were generated by inserting a homozygous point mutation into Eif2b5 (c.572G >A, p.Arg191His; $2 b 5^{h o}$ mice) and Eif2b4 (c.1450C>T, p.Arg484Trp; 2b4 ${ }^{\text {ho }}$ mice) (Figure 1, A and B). These mutations correspond to c.584G>A, p.Arg195His in EIF2B5 (23) and c.1447C >T, p.Arg148Trp in EIF2B4 (7), respectively, in humans, in whom they are associated with the most severe forms of VWM. The first mutation causes Cree encephalopathy, a VWM variant with onset soon after birth and death before 2 years of age (23). Patients homozygous for the second mutation have a neonatal presentation and die within a few months (7). Doubletransgenic animals with 1 homozygous mutation in Eif2b4 or Eif2b5 and 1 heterozygous mutation in the other IF2B gene showed similar disease severity with respect to disease course, lifespan, and pathology and were grouped ( $2642 b 5^{\text {he/ho }}$ mice). Animals homozygous for the mutation in both genes are referred to as $2 b 4^{h o} 2 b 5^{h o}$ mice.

Mutant strains developed a neurological phenotype with the following order of increasing severity: $2 b 4^{h o}, 2 b 5^{h o}, 2 b 42 b 5^{h e / h o}$, 
A

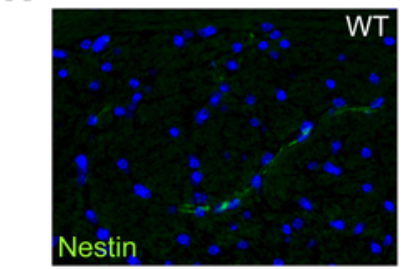

B

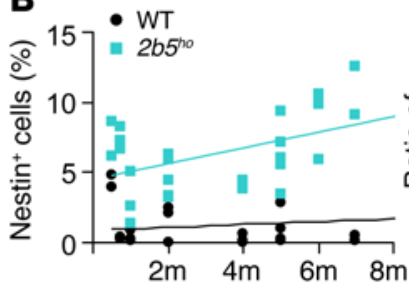

E

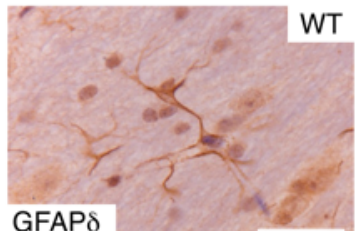

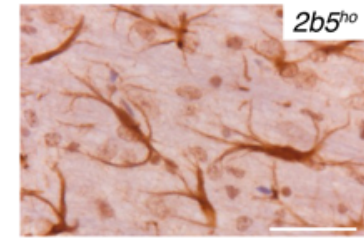

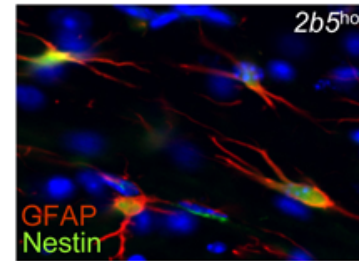

D
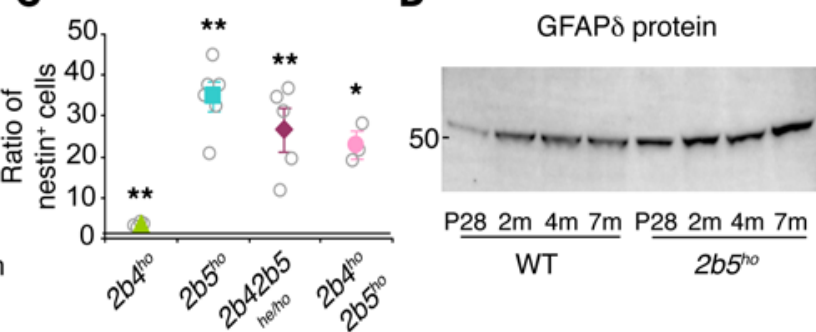

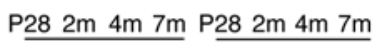
WT $2 b 5^{\text {ho }}$

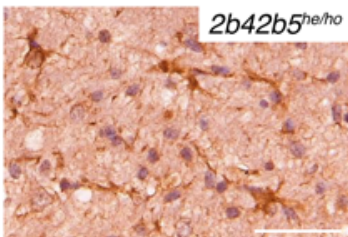

Figure 2. White matter astrocytes are immature and have abnormal morphology and intermediate filament composition. (A) Nestin-positive cells were present in the corpus callosum of 7-month-old $2 b 5^{\text {ho }}$ mice (middle), but not in that of 7-month-old WT mice (left). Double staining for GFAP confirmed that these cells were astrocytes (right). (B) The number of nestin-positive cells in the corpus callosum of $2 b 5^{\text {ho }}$ mice increased from P14 onward as the disease progressed ( $n=30$, WT mice; $n=21,2 b 5^{\text {ho }}$ mice). (C) In all VWM mutant mice, the number of nestin-positive cells in the corpus callosum was significantly increased ( $n=16,19$-month-old $2 b 4^{\text {ho }}$ mice; $n=6,4$-month-old $2 b 42 b 5^{\text {he/ho }}$ mice; $n=3, P 212 b 42 b 5^{\text {ho }}$ mice). (D) GFAP $\delta$ protein levels were increased in forebrain lysates from $2 b 5^{\text {ho }}$ mice at all ages examined. (E) Staining for GFAP $\delta$ showed increased immunoreactivity in white matter astrocytes from mutant mice compared with those from WT mice. (A and $\mathbf{E}$ ) Scale bars: $50 \mu \mathrm{m}$. (B and $\mathbf{C}$ ) ${ }^{*} P<0.05$ and ${ }^{*} P<0.01$ by Mann-Whitney $U$ test. Each data point in $\mathbf{B}$ indicates 1 mouse with a trend line; data points in $\mathbf{C}$ represent the ratio of mutant over WT, with the solid data point indicating the mean ratio of mutant over WT \pm SEM. Immunostainings are representative of at least 3 experiments.

and $2 b 4^{h o} 2 b 5^{h o}$. The $2 b 5^{h o}$ mice with intermediate disease severity were analyzed in detail and compared with less and more severely affected strains. The $2 b 4^{h o} 2 b 5^{h o}$ mice were troublesome to breed and only used for key experiments. All animals were analyzed at the disease endpoint. Additionally, $2 b 5^{h o}$ and $2 b 4^{h o}$ mice were analyzed throughout the disease course to study the development of VWM pathology. The number and age of VWM and WT animals used for the different experiments are provided in Supplemental Table 1 and in the Methods.

VWM mutants display growth restriction and variably severe neurological dysfunction. All mutants had lower body weights than did their WT littermates (Supplemental Figure 1A; supplemental material available online with this article; doi:10.1172/ JCI83908DS1) and developed progressive gait ataxia (Supplemental Figure 1B and Supplemental Videos 1-3). These mice also had sporadic epileptic seizures.

Analysis of spontaneous behavior in an automated home cage showed that $2 b 5^{h o}$ mice displayed longer arrests at 2 and 5 months and reduced activity per time interval with respect to the dark/ light phase at 5 months (Supplemental Table 2 and Supplemental Figure 1, C-E) as compared with that observed in WT mice. Behav- ioral tests indicated motor deficits at 5 months, with reduced grip strength $[n=15, t(12.98)=2.32, P=0.04$; Supplemental Figure 1F]; increased latency $[n=15, t(9.92)=-4.88, P<0.01$; Supplemental Figure 1G; Supplemental Table $3]$; and an increased number of foot slips $[n=15, t(8.98)=-3.4, P<0.01$; Supplemental Figure $1 \mathrm{H}$ ] when traversing a narrow beam compared with WT mice. The $2 b 5^{\text {ho }}$ mice died by 7 to 10 months of age (Figure 1C).

The $2 b 4^{h o}$ mice had onset of similar clinical features around 7 months of age and survived until 18 to 20 months of age. The $2 b 42 b 5^{\text {he/ho }}$ mice had disease onset at 6 weeks and an average lifespan of 4 to 5 months. The $2 b 4^{h o} 2 b 5^{h o}$ mice showed disease signs from P10 and survived less than 3 weeks (Figure 1C).

VWM mouse white matter shows perturbed myelination and progressive myelin vacuolization without microglia activation. In 1 - to 7 -month-old $2 b 5^{\text {ho }}$ mice, staining for the mature myelin proteins MBP and PLP showed the presence of myelin in all white matter structures. Increasing vacuolization was detected from 5 months of age (Figure 1D and Supplemental Figure 2A). Vacuoles were surrounded by myelin sheaths, and splitting occurred at the intraperiod line, indicating intramyelinic edema (Supplemental Figure 2, $\mathrm{A}$ and $\mathrm{B})$. MBP $(n=12, U=0, P<0.01)$ and MOG $(n=6, U=0, P=0.05)$ protein amounts and Mbp $[n=12, t(6.29)=2.52, P=0.04], P l p[n=12, t(10)=3.18, P=0.01]$, and $\operatorname{Mog}[n=12, t(10)=2.80, P<0.01] \mathrm{mRNA}$ levels were lower in $2 b 5^{h o}$ mice than in age-matched controls (Supplemental Figure 2, C-E). Total MBP expression was already reduced at P28, before the clinical onset [mRNA $n=4, t(1.81)=19.96, P<0.01$; protein $n=6$, $U=0, P=0.05]$; earlier than that, only a lower amount of the mature 14-kDa MBP isoform at P21 was found (Supplemental Figure 2D). $\mathrm{MBP}$ protein levels in $265^{h o}$ mice remained lower than in controls at all ages, but did not show a clear decrease over time (Supplemental Figure 2D). mRNA levels of oligodendrocyte-lineage transcription factor 2 (Olig2) and the OPC-specific marker Pdgfra, as well as total numbers of Nkx2.2- and Pdgfra-positive cells were unchanged (Supplemental Figure 2F and Supplemental Table 4). By contrast, the number of mature oligodendrocytes, as determined by Plp expression, was decreased $[n=11, t(9)=16.14, P<0.001$, Supplemental Figure $2 \mathrm{G}]$. Oligodendrocyte morphology was normal.

$2 b 4^{h o}$ mice showed a slight decrease in MBP protein at 19 months, while PlpmRNA levels were already significantly decreased at 7 months $[n=4, t(1)=14.67, P=0.04$; Supplemental Figure $2 \mathrm{E}$ and Supplemental Table 4]. Their white matter contained no vacuoles. 
A
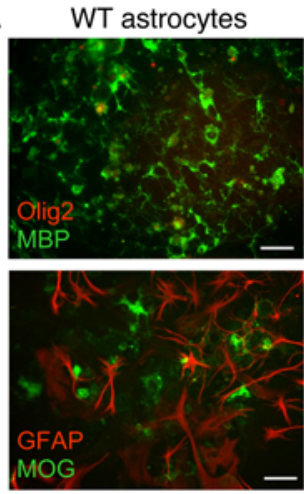

B

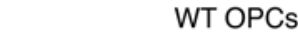

WT OPCs
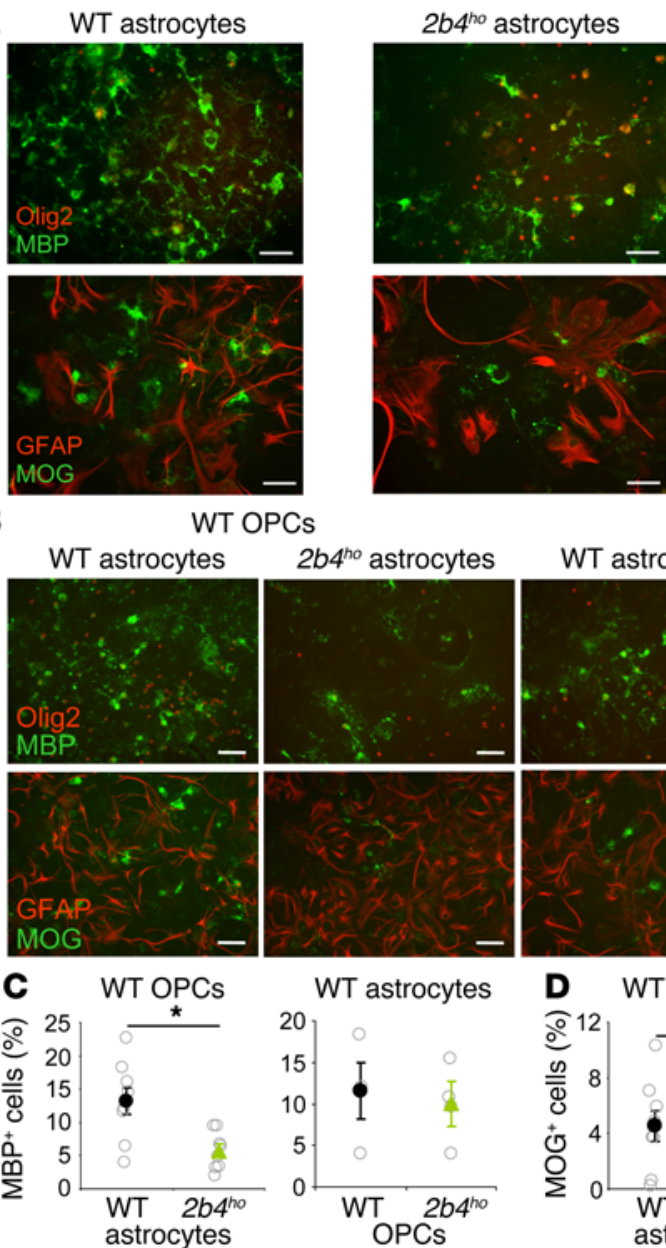
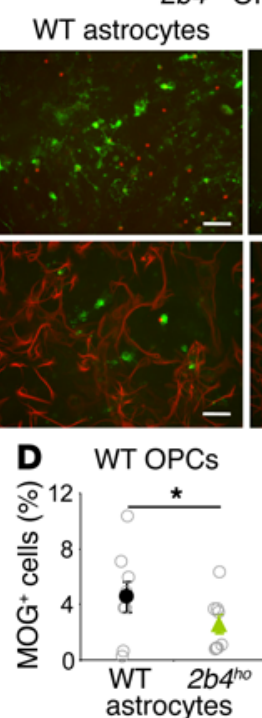

$2 b 5^{\text {ho }}$ astrocytes
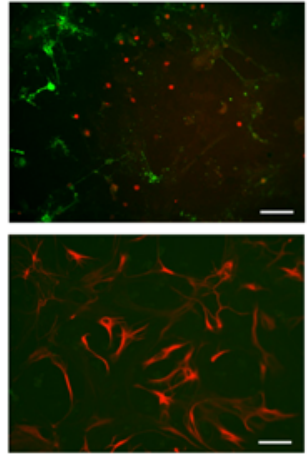

$2 b 4^{\text {ho }}$ OPCs

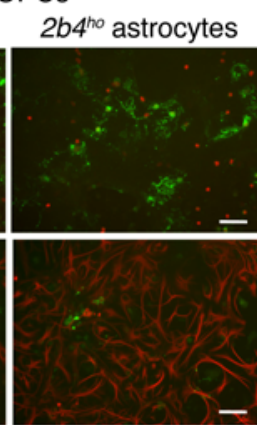

WT astrocytes

8

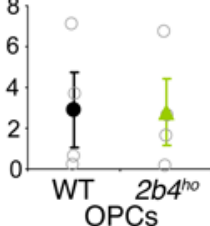

Figure 3. VWM astrocytes inhibit OPC maturation in vitro. (A) Compared with cocultures of WT astrocytes and WT OPCs (left), cocultures of $2 b 4^{\text {ho }}$ astrocytes and WT OPCs (middle) showed a decrease in MBP- and MOGpositive cells. Cocultures of $2 b 5^{\text {ho }}$ astrocytes and WT OPCs showed the lowest number of MBP- and MOG-positive cells (right). (B) Lower numbers of MBP- and MOG-positive cells were present in cocultures with $2 b 4^{\text {ho }}$ astrocytes, independent of the OPC genotype (WT or $2 b 4^{\text {ho }}$ ). No differences were seen between cultures with WT astrocytes and WT or $2 b 4^{\text {ho }}$ OPCs. Scale bars: $50 \mu \mathrm{m}$ (A and B). (C) The number of MBP-positive cells was significantly decreased in cocultures with $2 b 4^{\text {ho }}$ astrocytes $(n=8)$. There were no significant differences between cultures with WT or $2 b 4^{\text {ho }}$ OPCs $(n=6)$. (D) A similar pattern was observed for MOG-positive cells. (C and D) Data points indicate 1 experiment, with the solid data point indicating the mean \pm SEM. ${ }^{*} P<0.05$, by paired-samples $t$ test. Immunostainings are representative images of at least 3 experiments.
The 4-month-old $2 b 42 b 5^{\text {he/ho }}$ mice showed a paucity of myelin with pronounced vacuolization (Figure 1D and Supplemental Figure 2, A and B). Levels of MBP protein and Mbp mRNA $[n=4, t(1)$ $=4.86, P=0.04], P l p[n=4, t(1)=20.86, P=0.03]$, and $M o g[n=4$, $t(2)=11.31, P<0.01]$ were markedly lower than levels in WT mice (Supplemental Figure 2, D and E). Pdgfr $\alpha$ mRNA-expressing cells were increased, although not significantly (Supplemental Figure $2 F)$. P21 $2 b 4^{h o} 2 b 5^{h o}$ mice showed the most pronounced decrease in MBP immunoreactivity and the greatest degree of myelin vacuolization, with a significant increase in Pdgfr $\alpha$ mRNA-expressing cells $[n=6, t(4)=-7.03, P<0.01$; Figure 1D, Supplemental Figure $2 \mathrm{~F}]$. In both $2 b 42 b 5^{h e / h o}(n=9, U=0, P=0.014)$ and $2 b 4^{h o} 2 b 5^{h o}$ ( $n=6, U=0, P=0.046$ ) mice, the number of $P l p$-positive mature oligodendrocytes was decreased (Supplemental Figure $2 \mathrm{G}$ ). In all mice, oligodendrocyte morphology was normal.

An Iba1 stain was used to investigate microglia activation in $2 b 5^{\text {ho }}$ mice. Iba1-positive activated microglia were sparse in the white matter, far below the activation level observed in mice with experimental autoimmune encephalomyelitis (provided by Anne-Marie van Dam; data not shown). The Iba1 staining showed no macrophages.

In summary, in $2 b 5^{\text {ho }}$ mice, the total MBP protein and $M b p$ mRNA levels were decreased at P28, while the mature MBP $14-\mathrm{kDa}$ isoform levels were already decreased at P21, indicating that myelin deposition is deficient and myelin maturation is already delayed before clinical disease onset. In older $2 b 5^{\text {ho }}$ mice, the numbers of Plp-positive oligodendrocytes were lower, and expression of all mature myelin proteins was decreased, with no clear signs of myelin loss. A similar decrease in myelin proteins and mature oligodendrocytes was observed in the other VWM mutants. Additionally, the white matter of the most severely affected mice $\left(2 b 4^{h o} 2 b 5^{h o}\right.$ mice and, to a lesser degree, $2 b 42 b 5^{h e / h o}$ mice) contained increased OPC numbers. Progressive myelin vacuolization was observed, which correlated with disease severity, genotype, and disease stage. These results indicate deficient myelin formation, maturation, and maintenance in VWM mice.

Small-caliber axons and scattered axonal swellings are observed in VWM mice. In $2 b 5^{\text {ho }}$ mice, staining against SMI 32 and $\beta$ APP, which highlighted the impaired axonal cytoskeletal architecture and transport, showed a few scattered positive axonal swellings at 7 months only. Electron microscopic analysis of the corpus callosum at 7 months revealed a decreased mean axonal diameter $(n=572$, $U=10135, P<0.01)$, with an increased percentage of axons smaller than $0.6 \mathrm{~mm}$ in diameter $\left[n=192, X^{2}(5)=86.8, P<0.01\right.$; Figure $\left.1 \mathrm{E}\right]$. The ratio of axon/outer fiber diameter ( $g$ ratio) was significantly lower in $2 b 5^{h o}$ mice than in controls $(n=572, U=9096, P<0.01$; Supplemental Figure $2 \mathrm{H}$ ), also after correction for the smaller axonal diameter. A smaller axonal diameter, a higher percentage of small-caliber axons, and a significantly reduced $\mathrm{g}$ ratio were also detected in 4-month-old $2 b 42 b 5^{\text {he/ho }}$ mice $[n=530, U=11434.5$, 
A

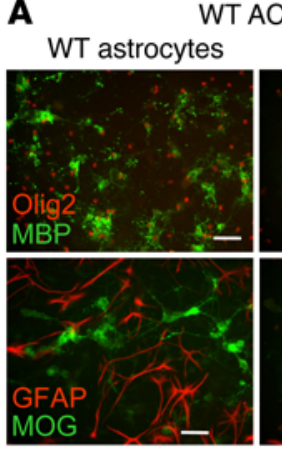

B

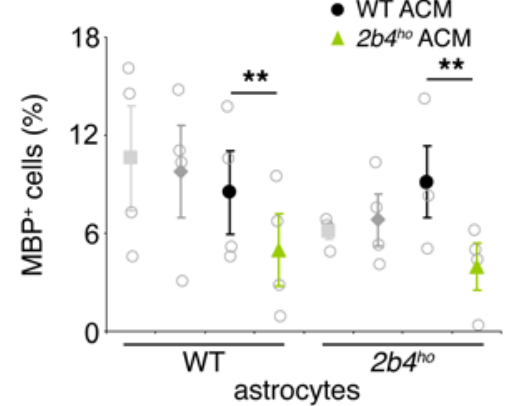

D

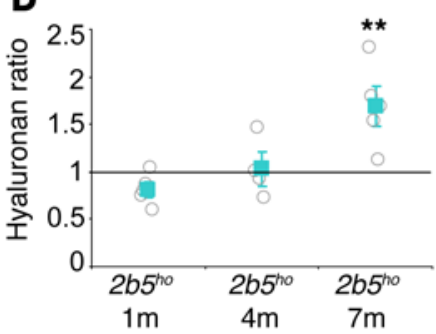

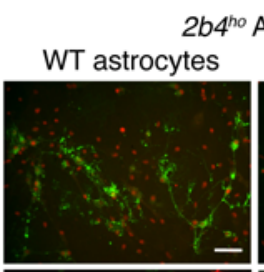

ACM
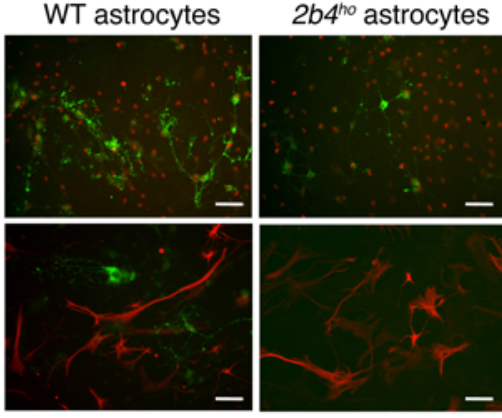

.

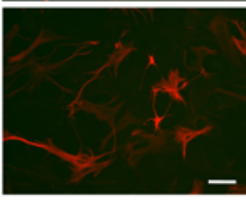

wk refresh

$\checkmark$ d refresh

- WT ACM
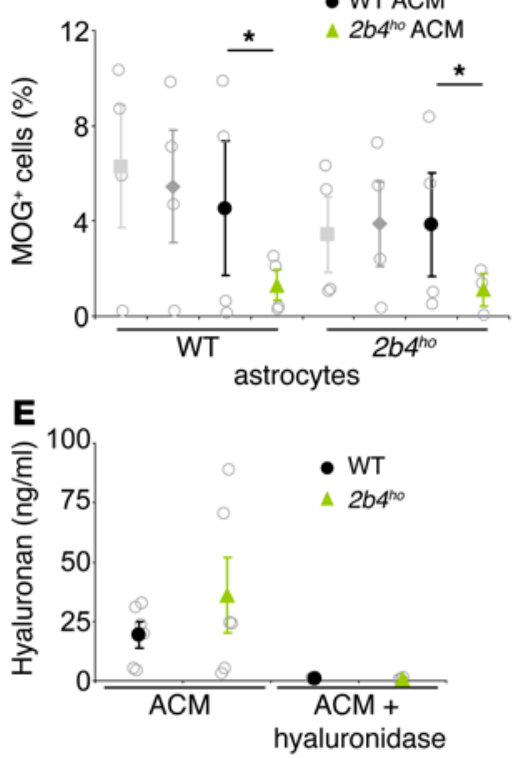

Figure 4. WT ACM rescues OPC maturation. (A) Immunostaining for olig2, MBP, GFAP, and MOC showed decreased immunoreactivity of MBP and MOC in the cocultures with $2 b 4^{\text {ho }}$ ACM, but not with WT ACM. Scale bars: $50 \mu \mathrm{m}$. (B and $\mathbf{C})$ The numbers of MBP- (B) or MOG-positive (C) cells were significantly lower in cocultures with WT and $2 b 4^{\text {ho }}$ astrocytes when grown in $2 b 4^{\text {ho }} \mathrm{ACM}$, but the numbers increased with exposure to WT ACM ( $n=4$ for all). (B and C) "wk refresh" indicates refreshment of the medium once per week, as was done for all other cocultures; "d refresh" indicates a daily refreshment of the medium, as a control for the daily refreshment in the conditioned medium experiments. (D) The amount of hyaluronan was significantly increased in 7-month-old $2 b 5^{\text {ho }}$ mice, but not in 1-month-old or 4-monthold $2 b 5^{\text {ho }}$ animals ( $n=5$ for all). (E) ACM of WT and $2 b 4^{\text {ho }}$ mice showed no significant differences in hyaluronan levels, although in two $2 b 4^{\text {ho }}$ mutant mouse ACM samples, the hyaluronan levels were greatly increased. After treatment with hyaluronidase, no hyaluronan signal was detected by ELISA in any sample ( $n=6$ for all). (B, C, and E) Data points indicate 1 experiment, with solid data points indicating the mean \pm SEM. (D) Data points represent the ratio of mutant over WT, with solid data points indicating the mean ratio of mutant over WT \pm SEM. ${ }^{*} P<0.05$ and ${ }^{* *} P<0.01$, by paired-samples $t$ test (B and C), Student's $t$ test (D), and Mann-Whitney $U$ test (E). Immunostainings are representative images of at least 3 experiments.
$P<0.01 ; n=199, X^{2}(5)=68.64, P<0.01$ and $n=530, U=15738$, $P<0.01$, respectively; Figure 1E, Supplemental Figure $2 \mathrm{H}]$. In all mutants, the smaller axons appeared ultrastructurally normal.

White matter astrocytes in VWM are immature and have abnormal morphology and intermediate filament composition. To assess the maturation status of mutant white matter astrocytes, we double stained for GFAP and the immature intermediate filament protein nestin. The number of GFAP and nestin double-positive cells in the white matter was higher in $2 b 5^{h o}$ mice than in WT animals as early as P14, and the number increased further after clinical onset $\left(n=39, U=19, P<0.01 ; R^{2}=0.574 ;\right.$ Figure $\left.2, \mathrm{~A}-\mathrm{C}\right)$. Nestin mRNA levels were also increased $[n=12, t(10)=2.24, P=0.05$; Supplemental Figure 3A].

Nestin-positive astrocytes had thick, coarse processes with strong GFAP $\delta$ immunolabeling (Figure 2, D and E). An abnormal morphology was observed with staining for both GFAP and the membrane protein GLT1 (not shown). Total levels of Gfap mRNA and GFAP protein were unchanged (Supplemental Table 4). GFAP $\delta$ protein levels were significantly increased $[n=6, t(4)=$ -3.892, $P=0.02$; Figure 2D and Supplemental Figure $3 \mathrm{~B}]$, as was the GFAP $\delta / G F A P$ ratio $(n=6, U=0, P=0.05$; Supplemental Figure 3C). Phosphorylated STAT3 (p-STAT3), a transcriptional regulator that is increased during classic astrogliosis (24), was not upregulated at 7 months (data not shown). Overexpression of nestin and
GFAP $\delta$ in the absence of upregulation of total GFAP and p-STAT3 indicates that $2 b 5^{h o}$ white matter astrocytes are immature and reactive gliosis is compromised.

Significantly increased numbers of GFAP/nestin double-positive astrocytes were also found in the white matter of $2 b 4^{\text {ho }}$ mice from 2 months of age onward $(n=40, U=43, P<0.01)$, of 4-month-old $2 b 42 b 5^{\text {he/ho }}$ mice ( $\left.n=30, U=10, P<0.01\right)$, and of P21 $2 b 4^{h o} 2 b 5^{h o}$ mice $[n=8, t(2.09)=-8.08, P=0.01]$ (Figure $2 C$ ). In $2 b 4^{h o}$ animals, analyzed at multiple time points, the progressively higher numbers of nestin-positive astrocytes paralleled the clinical worsening (not shown), as was the case in $2 b 5^{h o}$ mice. Nestin mRNA levels were significantly increased in $2 b 42 b 5^{\text {he }}$ ho mice compared with levels in WT mice $[n=4, t(1.22)=-13.91, P=0.03$; Supplemental Figure 3A]. Many nestin-positive astrocytes had aberrant morphology and stained strongly for GFAP $\delta$ (Figure 2E).

The hippocampus and cerebral cortex of all mutant mice showed mild gliosis, the expected morphological correlate of epilepsy. In these areas, reactive protoplasmic astrocytes had normal morphology with delicate, branched cell processes and GFAP $\delta$ immunoreactivity comparable to what was seen in the WT animals (Supplemental Figure 3D).

In summary, all VWM mutant mice had abnormal white matter astrocytes, even $2 b 4^{h o}$ mice that lacked clear myelin pathology. The number of nestin-positive immature astrocytes increased before 
A

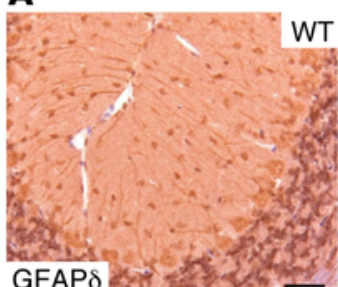

B
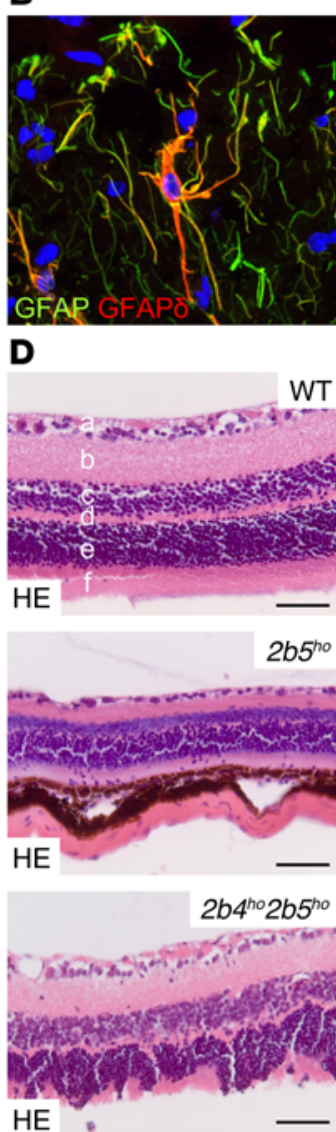
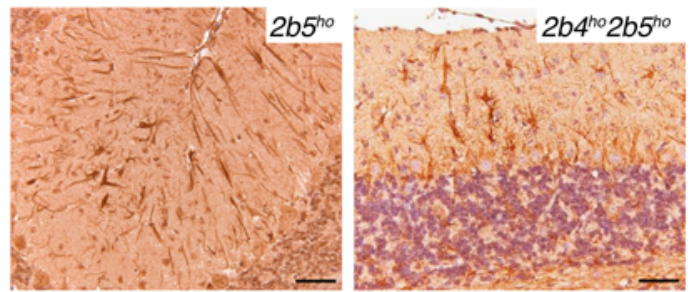

c

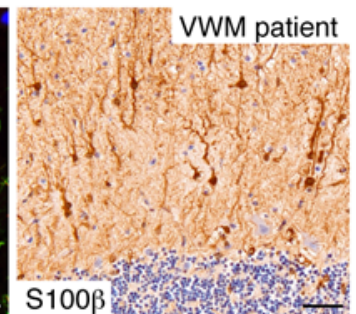

E

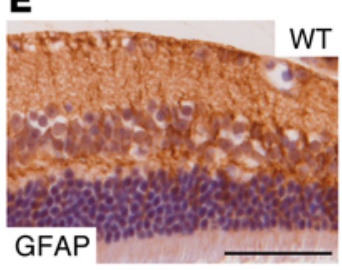

$\mathbf{F}$

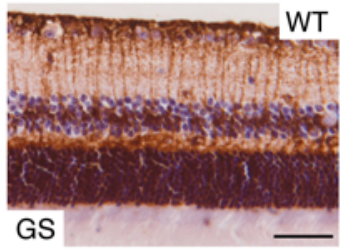

$2 b 5^{\text {ho }}$
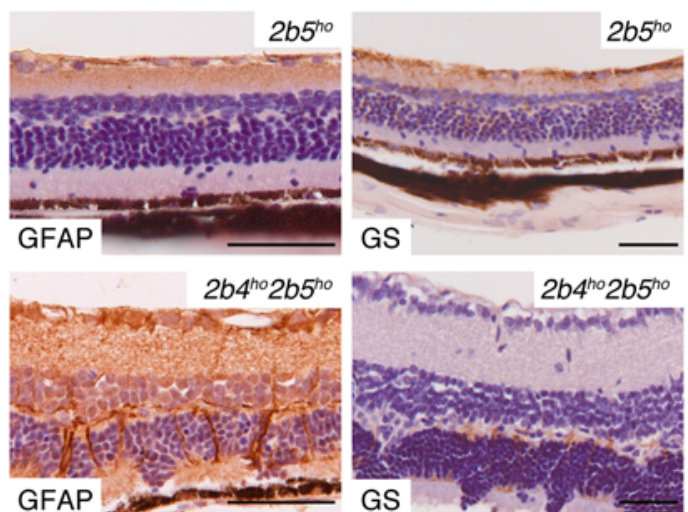

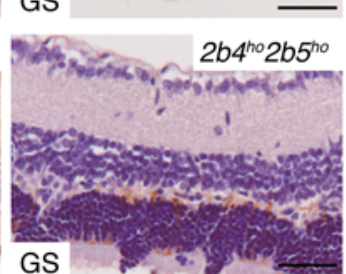

Figure 5. Astrocyte pathology outside the brain white matter. (A) In the cerebellum of 7-month-old $2 b 5^{\text {ho }}$ and P21 $2 b 42 b 5^{\text {ho/ho }}$ mice, Bergmann glia overexpressed GFAP $\delta$, with displacement of the cell bodies to the molecular layer and abnormal morphology with short, thick cell processes. (B and C) A similar Bergmann glial pathology was also present in VWM patients as shown in double stainings for GFAP and GFAP $\delta$ (B) and S100 $\beta$ (C). (D) Histology of WT retina showed linear organization of the retinal layers. a, ganglion cell; b, inner plexiform; c, inner nuclear; d, outer plexiform; e, outer nuclear; f, photoreceptor. VWM mutant retinae showed increasing laminar disorganization, with ectopic neurons in the plexiform layers and severe displacement of outer nuclear cells in the P21 $2 b 4^{\text {ho }} 2 b 5^{\text {ho }}$ animals (bottom). (E) Staining against GFAP showed decreased immunoreactivity in the outer plexiform layer of retinae from $2 b 5^{\text {ho }}$ mice, whereas the $\mathrm{P} 212 b 4^{\text {ho }} 2 b 5^{\text {ho }}$ mouse retinae contained Müller cells with coarse processes spanning across the outer nuclear and photoreceptor layer. (F) Staining against glutamine synthetase (CS) showed markedly reduced immunoreactivity in the Müller glia of the $2 b 5^{\text {ho }}$ and $2 b 4^{\text {ho }} 2 b 5^{\text {ho }}$ mutants. Scale bars: $50 \mu \mathrm{m}$ (A, C, E, and F); $20 \mu \mathrm{m}$ (B); $200 \mu \mathrm{m}$ (D). Immunostains are representative images of at least 3 experiments. other histologic abnormalities, such as deficient myelin deposition and myelin vacuolization, and long before the onset of the clinical phenotype. The numbers of GFAP/nestin double-positive immature astrocytes correlated with disease severity and progression.

VWM astrocytes inhibit OPC maturation by secreted factors. To assess whether astrocytes have an impact on OPC maturation in VWM, we cultured OPCs on a confluent, enriched astrocyte monolayer. Because of the lower breeding efficiency of $2 b 5^{h o}$ mice, $2 b 4^{\text {ho }}$ animals were used for most experiments; the reported cell counts and statistical analyses refer to cultures from $2 b 4^{h o}$ and WT animals. No differences in numbers of GFAP- and olig2-positive cells or Gfap and Olig2 mRNA levels were observed between WT and VWM astrocytes (data not shown). Stains for the myelin proteins MBP and MOG were used as markers of oligodendrocyte maturation. MOG is expressed by mature oligodendrocytes slightly later than MBP, allowing the identification of maturation defects that arise after MBP expression. In cocultures of $2 b 4^{\text {ho }}$ astrocytes and WT OPCs, the number of MBP- $(n=16, Z=-2.38, P=0.02)$ and MOG-positive cells $(n=16, Z=-2.25, P=0.03$ ) (Figure $3 \mathrm{~A}$ ) and the levels of Mbp and Mog mRNA (Supplemental Table 4) were decreased compared with what was observed in cocultures of WT astrocytes and WT OPCs. The number of MBP- and MOG-positive mature oligodendrocytes was lower in $2 b 5^{h o}$ and $2 b 4^{h o}$ cocultures than in WT cocultures (Figure 3A), indicating that VWM astrocytes from both mutants inhibited OPC maturation in vitro. By contrast, cocultures of WT astrocytes with $2 b 4^{\text {ho }}$ or WT OPCs showed no difference in the number of cells positive for $\operatorname{MBP}[n=16, t(7)=0.89$, $P=0.41] ;$ MOG $[n=16, t(7)=0.1, P=0.92]$; and olig2 $[n=12, t(5)=$ $-0.05, P=0.97$ ] (Figure 3, B-D, and Supplemental Figure 4, A and B) indicating that, in the absence of mutant astrocytes, $2 b 4^{\text {ho }}$ OPCs are capable of normal maturation in vitro.

To determine whether the observed OPC maturation defect is mediated by secreted factors, cocultures were exposed to astrocyte-conditioned medium (ACM) collected from WT or $2 b 4^{\text {ho }}$ astrocytes. Cocultures with $2 b 4^{\text {ho }}$ ACM showed a significantly lower number of MBP- and MOG-positive cells than did cultures with WT ACM $[n=16, t(7)=4.94, P<0.01$ and $n=16, t(7)=3.12$, $P<0.02$, respectively; Figure $\left.4, \mathrm{~A}^{-} \mathrm{C}\right]$. In cocultures of $2 b 4^{h o}$ astro- 
cytes with WT OPCs, oligodendrocyte maturation was rescued by WT ACM. The numbers of GFAP- and olig2-positive cells were similar in all tested conditions (Supplemental Figure 4, C-F, and Supplemental Table 4), indicating no difference in the survival of OPCs or astrocytes. The cocultures and ACM experiments show that $2 b 4^{h o}$ astrocytes inhibit OPC maturation through factors secreted in the medium.

One factor known to inhibit OPC maturation is hyaluronan (22), which is increased in the white matter of VWM patients (21). In mouse forebrain lysates, hyaluronan was significantly increased in 7-month-old $2 b 5^{h o}[n=10, t(8)=-3.46, P<0.01]$ and P21 $2 b 4^{h o} 2 b 5^{h o}[n=6, t(4)=-2.77, P=0.05]$ (Supplemental Table 4) mice compared with age-matched controls. Hyaluronan was not significantly increased in 1 - or 4 -month-old $2 b 5^{\text {ho }}$ mice relative to levels in age-matched controls, but the relative level rose with age between 1 and 7 months $[F(1,5)=37.51, P=0.002$; Figure 4D]. Staining for the major hyaluronan-synthesizing enzyme HAS2 in different mutants showed increased immunoreactivity in the 9-month-old $2 b 5^{h o}$ and P21 $2 b 4^{h o} 2 b 5^{h o}$ mice compared with age-matched controls, matching the results of the ELISA (Supplemental Figure 5, A and B). HAS2-positive cells had the morphology of astrocytes.

In ACM of $2 b 4^{\text {ho }}$ astrocytes, hyaluronan levels were variable and, on average, not significantly increased (Figure 4E). To further assess the impact of hyaluronan on OPC maturation, ACM pretreated with hyaluronidase was applied to cultures of WT OPCs. Hyaluronidase pretreatment of both WT and $2 b 4^{h o} \mathrm{ACM}$ increased the number of MBP-positive cells $[n=12, t(5)=2.66, P=0.05$; Supplemental Figure $5 \mathrm{C}$ ], while the number of olig2-positive cells was unchanged (Supplemental Table 4). There were no differences in the improvement in OPC maturation upon hyaluronidase treatment between $2 b 4^{h o}$ and WT ACM (Supplemental Figure 5D). No correlation between the level of hyaluronan and OPC maturation was observed in the vehicle-treated cultures (data not shown).

Bergmann glia are abnormal in VWM mice. In the cerebellar cortex of 5- and 7-month-old $2 b 5^{\text {ho }}$ mice, increasing numbers of Bergmann glia were mislocalized to the molecular layer and had abnormally oriented, thicker, and more intensely GFAP $\delta$-immunoreactive processes than did glia from WT mice (Figure $5 \mathrm{~A}$ ). No ectopic Bergmann glia were seen in younger $2 b 5^{h o}$ mutants. The $2 b 5^{h o}$ cerebellar cortex was otherwise normal (Supplemental Figure 6A).

Strongly GFAP $\delta$-immunopositive ectopic Bergmann glia were also seen in the other mutants' cerebella. Especially in P21 $2 b 4^{h o} 2 b 5^{h o}$ mice, virtually all Bergmann glia were mislocalized to the molecular layer and were GFAP $\delta$ immunopositive (Figure 5A and Supplemental Figure 6B).

These findings in mice prompted a reexamination of the cerebella of 12 VWM patients of different ages. Staining against GFAP and $S 100 \beta$ revealed ectopic Bergmann glia in the cerebellar cortex of all patients (Figure 5, B and C), a phenomenon that was most prominent in infantile- and early childhood-onset cases. Also in patients, ectopic Bergmann glia were markedly GFAP $\delta$ immunopositive (Figure 5B). As in mice, the cerebellar cortex of VWM patients was otherwise normal (Supplemental Figure 6, C and D).

The retina is disorganized in VWM mice. Antenatal-onset VWM is associated with cataract (7). None of the mutants had cataract, but all showed signs of retinal laminar disorganization (Figure 5,
D-F). Retinal changes consisted of uneven margins of the inner and outer nuclear layers with a thinned inner plexiform layer, ectopic inner nuclear cells, and displaced granule cells from the outer nuclear layer to the photoreceptor layer. These findings were most pronounced in the 3-week-old $2 b 4^{h o} 2 b 5^{h o}$ mice (Figure 5, D-F), in which staining for glutamine synthetase, normally present in Müller glia, was virtually negative. In mutant mice, GFAP staining showed that Müller glia had thick processes crossing the inner and outer nuclear layers and reaching the inner limiting membrane and the photoreceptor layer (Figure 5D).

No involvement of extracerebral organs in VWM mice. Histological analysis of internal organs, skeletal muscle, and peripheral nerves showed no differences between mutant and WT animals (data not shown).

\section{Discussion}

Since we identified the genes that were mutated in VWM more than a decade ago $(2,3)$, the pathophysiology of this disease has been addressed at different levels, including genetic, biochemical, histopathologic, and immunohistochemical, with limited success $(5,10-14,20,21,25,26)$. In the present study, we address the cellular pathomechanisms of VWM.

VWM mutant mice recapitulate the human disease and reveal that astrocytes are central in the cellular pathomechanisms. In VWM patients, the early white matter disease course is only documented by MRI because of the lack of brain tissue from this stage. In young presymptomatic VWM patients, MRI shows mild signal abnormalities in the subcortical white matter, indicating deficient myelination, and more prominent signal abnormalities in the periventricular white matter compatible with myelin vacuolization and loss $(5,27)$. Over time, MRI shows extension of the prominent white matter abnormalities and evidence of increasing rarefaction and cystic degeneration. At autopsy at end-stage disease, the white matter is variably cavitated, with areas of loss of all structures, including myelin and axons. The relatively intact white matter shows vacuolization, a paucity of myelin, and increased numbers of OPCs, which proliferate $(17,19,20)$ but do not develop into mature myelin-forming cells $(20,21)$. A small proportion of the oligodendrocytes has an abnormal, foamy appearance $(17,19$, 28). Our VWM mutant mice confirmed the deficient oligodendrocyte maturation and myelin deposition as well as the myelin vacuolization. These features were already present at the presymptomatic stage and increased in severity with disease progression. The number of mature oligodendrocytes was decreased, and, in the most severe mutants, the number of OPCs was increased. No foamy oligodendrocytes were detected in VWM mice. The exact cause of the myelin vacuolization in VWM patients and mutant mice is not known. Astrocytes play a key role in maintaining and regulating brain ion and water homeostasis (29). Defects in the astrocyte-specific MLC1, GlialCAM, and ClC2 proteins as well as astrocyte-expressed connexins cause myelin vacuolization (29). It is tempting to speculate that astrocytic dysfunction also causes the myelin vacuolization seen in VWM. Functional studies are needed to confirm this hypothesis.

In human VWM disease white matter, axonal swellings and loss are only observed in severely affected areas; axons in relatively preserved areas are microscopically intact $(1,16)$. Reduced axonal 
diameters have been reported (27). VWM mice have a higher proportion of small-caliber axons than do WT animals (present study; ref. 30). Scattered axonal swellings are only detected in the oldest mutants with advanced disease, but the axonal cytoskeleton appears otherwise intact. The axonal changes are most likely secondary to myelin pathology (31-33).

White matter astrocytes in human VWM have abnormal morphology with blunt processes $(16,20)$. Their immunohistochemical profile indicates immaturity rather than reactive gliosis (20, $21,24)$. Immature astrocytes are most preponderant in the most severely affected white matter areas (21). In contrast to one study indicating a compromised generation of human VWM astrocytes in vitro (18), we found increased proliferation both in vitro and in situ $(20,21)$. In the present study, VWM mutant mice had abnormal white matter astrocytes, even the $2 b 4^{h o}$ mice, which lack clear myelin or oligodendrocyte pathology. Neither in vitro nor in vivo astrocyte generation was compromised in any of the VWM mice. White matter astrocytes had abnormally blunt processes and a immunohistochemical profile indicating arrested maturation rather than reactive gliosis, as deduced from the lack of upregulation of GFAP and p-STAT3. The number of immature astrocytes increased before other histologic abnormalities, long before clinical disease onset, and correlated with disease progression. These data indicate that white matter astrocytic abnormalities precede the involvement of oligodendrocytes and co-vary with the severity of the general white matter pathology.

Human VWM astrocytes overexpress GFAP $\delta$ (21). GFAP has different splice variants. GFAP $\alpha$ is the most abundant isoform in human and mouse brains $(34,35)$ and has the best intrinsic capacity to form cytoskeletal intermediate filaments, whereas increased GFAP $\delta$ yields condensed cytoskeletal networks (34). The stoichiometry of GFAP isoforms does not change during aging or reactive gliosis and disease $(34,36)$. In VWM patients, dysmorphic white matter astrocytes overexpress GFAP $\delta$, whereas total GFAP levels are unchanged $(20,37)$. The same occurs in VWM mouse astrocytes. VWM is the only disease known to show absolute overexpression of GFAP $\delta$ with unchanged total GFAP levels. The abnormal GFAP $\delta$ /GFAP ratio may have functional consequences for the cytoskeletal architecture of astrocytes, their ability to scar damaged tissue, and their interaction with other cell types, including oligodendrocytes (35).

Until now, abnormal astrocytes in VWM disease have only been reported within the brain white matter. Strikingly, in VWM mice, 2 additional astrocyte populations were found to be affected: Bergmann glia in the cerebellar cortex and Müller cells in the retina. Of note, Müller cells are affected in the retina in the absence of oligodendrocytes. These findings further support the assertion that astrocytes, rather than oligodendrocytes, are primarily affected in VWM disease.

Bergmann glia are located in the Purkinje cell layer and extend processes to the pial surface; these glial cells guide the migration of granule cells during development (38). In our VWM mice, Bergmann glia were displaced to the molecular layer and showed withdrawal of endfeet at the pial surface, increased process thickening, lateral branching, and strong GFAP $\delta$ immunoreactivity. These abnormalities became apparent with increasing disease severity. Consistent with this, cerebellar cortical development was unaf- fected and showed no granule cell migration defects or Purkinje cell abnormalities. Strikingly, we confirmed the same Bergmann glial pathology, not noted before, in VWM patients.

Müller cells are the main glia of the retina. They are located in the inner nuclear layer, and their processes span the retina (39). VWM mice show retinal disorganization with displacement of outer nuclear neurons to the photoreceptor layer. In $2 b 4^{h o} 2 b 5^{h o}$ mice, these changes are associated with abnormal Müller glia morphology, with thick coarse processes and loss of glutamine synthetase immunoreactivity. Retinal dysfunction has not been reported in VWM and is rarely investigated, because patients do not manifest clinical signs of retinal involvement. However, a retrospective inventory of available electroretinographic data in our patient database revealed that the patients studied had preservation of the a-wave and loss of the $b$-wave of the trace. These findings are indicative of a reduced activity of outer nuclear bipolar neurons, as observed in the case of Müller glia dysfunction with reduced glutamine synthetase activity (40), indicating that retinopathy is also part of the human VWM phenotype and related to Müller glia pathology.

The combined human and mouse data indicate that astrocytic abnormality is a core feature of VWM. To disentangle intrinsic and extrinsic influences on OPC maturation and confirm a primary role of astrocytes, we used cocultures with different combinations of WT or VWM astrocytes and OPCs and different ACMs. These studies confirm that the OPC maturation defect in VWM is not an intrinsic feature of oligodendrocyte-lineage cells, but rather is driven by astrocytic pathology. Our findings also indicate that the astrocytic inhibition of OPC maturation is mediated by secreted factors. While the maturation of VWM OPCs was normal in the presence of WT astrocytes, medium conditioned by VWM astrocytes inhibited OPC maturation also in the presence of WT astrocytes, and this maturation blockage was lifted by WT ACM. Many astrocyte-secreted factors influence OPC maturation and myelination (41-43). One such factor is hyaluronan (22). We recently showed that hyaluronan is increased in the white matter of deceased VWM patients and that hyaluronan levels correlate with severity of the white matter involvement at end-stage disease; earlier disease stages could not be investigated because of a lack of available brain tissue (21). In VWM mice, hyaluronan levels were normal in early stages and significantly increased over time, paralleling the increasing disease severity. These data suggest that hyaluronan plays a role in later and more severe disease stages, but does not cause the maturation defect observed in vitro. Our coculture results indicate that there must be additional, as-yet unidentified factors secreted by VWM astrocytes that inhibit OPC maturation.

Leukodystrophic mouse models. We believe that our VWM mouse model is unique, in that it recapitulates a degenerative leukodystrophy. While leukodystrophies characterized by developmental hypomyelination are recapitulated by mutant mice, e.g., jimpy (32) and shiverer (44) mice, mouse models for degenerative leukodystrophies are often unsuccessful (45-48). Major mouse-human interspecies differences, including lifespan, amount of cerebral hemispheric white matter, and differences in physiology and biochemistry, are limiting factors. Given the significant lifespan difference, it is not surprising that leukodystrophies with onset in or after childhood are typically not recapit- 
ulated in mutant mice (45-48). Additional manipulations may force mice to develop a phenotype that more closely matches that of the human disease, but these have the unwanted side effect of influencing the disease mechanisms (49-52). Geva et al. (30) previously generated a VWM mouse model by inserting Arg132His into eIF2B $\varepsilon$, a mutation that in the homozygous state in humans is associated with childhood-onset disease and death in adolescence. This mutant mouse had a normal lifespan, subtle motor impairment at most, and white matter abnormalities that only became manifest after experimental demyelination $(30,53)$. Using transgenic mice that allow activation of PERK specifically in oligodendrocytes, Lin et al. (52) concluded that PERK activation in oligodendrocytes plays a cell-autonomous role in VWM pathology. PERK activation decreases eIF2B activity via phosphorylation of the initiation factor eIF2, while in VWM, eIF2B activity is affected by mutations in eIF2B subunit genes and not invariably decreased (9). Our results, especially the coculture data, contradict the conclusion of Lin et al. (52).

To obtain valid pathophysiological insight, mouse models need to truly represent the human disease and be based on the same pathomechanisms. The 5-subunit complex eIF2B and its role in mRNA translation are highly conserved in all eukaryotes up to yeast (54), increasing the chance of a successful mutant mouse model if factors such as lifespan are taken into account. For this reason, we chose 2 mutations that both lead to early infantile fatal disease. We bred the 2 single-mutant mouse strains into double mutants, with the expectation of obtaining a more severe VWM variant. Patients generally do not have more than 2 mutations in eIF2B, although a very small proportion of patients do, while still having the typical VWM phenotype (55). Indeed, the phenotypes of the different mutants differ only in severity, indicating the same underlying pathomechanisms across the strains. The double mutants can therefore be considered representative of severe VWM.

A remaining potential concern is that mouse astrocytes are different from human astrocytes in many aspects, including size, protein expression, and calcium signaling $(36,56,57)$. It is therefore possible that the results obtained in mouse VWM astrocytes may only be partly applicable to human VWM astrocytes. However, given that the leukoencephalopathy in these mouse models is strikingly similar to that seen in human VWM, especially with regard to the phenotype of astrocytic abnormality and the astrocytic cell types affected, it can be concluded that VWM astrocytes are affected in a similar way in the 2 species, making the mouse models suitable for the study of human VWM pathophysiology. In order to dissect the exact roles of astrocytes and oligodendrocytes in VWM pathology in vivo, an interesting possibility would be to make conditional VWM mice, in which the mutation would only be present in oligodendrocytes or astrocytes.

Conclusion. VWM is a devastating disease that mainly affects young children and lacks effective therapeutic strategies. We provide the first confirmation to our knowledge that astrocytic dysfunction constitutes the basis of VWM pathology. Given these results, targeting astrocytes or factors secreted by astrocytes should be considered in future treatment strategies for VWM patients. In particular, modulation of hyaluronan levels to support OPC maturation could be considered, possibly in combination with cell-based therapies, to repopulate the white matter with healthy glia progenitors. Astrocytes are generally not considered to have a role in the pathophysiology of white matter disorders, but our study demonstrates that they should be.

\section{Methods}

Animals. We generated 2 mouse strains (background strain C57Bl/6J)

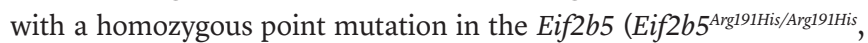
referred to as $2 b 5^{\text {ho }}$ mice) or Eif $2 b 4$ gene (Eif2b $4^{\text {Arg484Trp/Arg484Trp }}$, referred to as $2 b 4^{h o}$ mice). We selected mutations that are known to cause a severe variant of VWM in patients and developed 2 mouse models were later bred to generate heterozygous-homozygous and homozygous-homozygous double-mutant mice. Detailed information about the strategy used to generate the mutant strains is provided in Figure 1 and the Supplemental Methods.

Mutant and WT animals were weaned at P21 and had ad libitum access to food pellets and water. The animals were sacrificed at different ages for the studies planned, or when they became unable to take food or water or lost more than $15 \%$ of their body weight. The $2 b 5^{h o}$ mice were analyzed during development at weekly intervals (P0, P7, $\mathrm{P} 14$, and P28). Both $2 b 4^{h o}$ and $2 b 5^{h o}$ mice were also collected at regular intervals throughout their lifespan (Supplemental Table 1). A more detailed overview of the number of animals used in each experiment is provided in Supplemental Table 1.

Behavioral testing. At 2 and 5 months of age, the spontaneous behavior of ten $2 b 5^{h o}$ mice and ten WT male littermates was measured in an instrumented home cage (Noldus Information Technology) for 3 consecutive days as previously described (58). Spontaneous behavior testing gives a general indication of whether mice are behaving normally, but it is difficult to correlate the findings to a specific cellular defect. Motor behavior was tested with the grip strength meter, balance beam tests, and the paw-print test. Further details on behavior and motor tests are provided in the Supplemental Methods.

Astrocyte-OPC cocultures. Astrocyte-enriched cultures were obtained from the forebrains of E18 WT, $2 b 4^{h o}$, or $2 b 5^{h o}$ mice by papain digestion on a GentleMACS dissociator according to the manufacturers' protocol (Miltenyi Biotec). After isolation, astrocytes were cultured for 4 passages in astrocyte medium (DMEM/F12,10\% FBS, L-glutamine, and penicillin-streptomycin). Passaging was done by trypsin incubation for 5 to 10 minutes at $37^{\circ} \mathrm{C}$, after which cells were split 1:2 or 1:3 into a new flask. At passage 4 , cells were frozen and stored in liquid nitrogen until further use. WT and $2 b 4^{h o}$ astrocytes showed similar proliferation rates. Before starting a coculture, astrocytes were plated on a PLO-laminincoated plate in astrocyte medium at high density $\left(1 \times 10^{5}\right.$ cells in 1 well of an 8-well chamber slide) to form a confluent monolayer. Under these conditions, no or very low proliferation of the astrocytes was observed. Astrocytes were given a week to recover from the freezing before $1 \times 10^{5}$ OPCs were added to each well as described below. At this point, astrocyte cultures were devoid of MBP-immunopositive cells (Supplemental Figure 7A) and had a very low number of olig2-immunopositive cells $(\sim 1 \%)$. A small number of microglia were present in these cultures $(\sim 7 \%$, Supplemental Figure 7, B and C). Neurons were not observed.

OPCs were isolated by papain digestion of E18 mouse forebrain on the GentleMACS dissociator according to the manufacturers protocol (Miltenyi Biotec). After isolation, cells were plated overnight on a nonadherent culture plate in M41 medium (described as DMEM/F12/N1 in Sim et al., ref. 59) supplemented with $20 \mathrm{ng} / \mathrm{ml}$ basic FGF (bFGF). The following day, cells were sorted for PDGF $\alpha$ R expression to spe- 
cifically isolate OPCs. Sorting was done according to the manufacturers protocol (Miltenyi Biotec), with the use of a biotinylated PDGF $\alpha \mathrm{R}$ $\mathrm{Ab}$ (anti-CD140a, $1.5 \mu \mathrm{l}$ per $1 \times 10^{7}$ cells; eBioscience) and anti-biotin microbeads (Miltenyi Biotec) to label the target cells. After sorting of OPCs, the astrocyte plate was washed with PBS, and OPCs were plated on astrocytes in M41 medium. When OPCs were used for monocultures, the cells were resuspended in M41 medium and plated at a density of $3 \times 10^{5}$ cells per well on PLO/laminin-coated 8-well chamber slides. OPCs in culture without astrocytes showed lower maturation $(\sim 2 \%)$ and survival than did OPCs cultured with astrocytes or ACM.

The medium of the astrocyte-OPC cocultures was refreshed after 3 days. Cultures were maintained for 1 week, at which point the inhibition and promotion of OPC maturation by interventions would be measurable (Supplemental Figure 7D). After 1 week, cultures were either fixed with $2 \%$ paraformaldehyde (PFA) for 20 minutes for immunostaining or lysed with TRIzol (Life Technologies) for 5 to 10 minutes at room temperature (RT) for mRNA isolation. For each experiment, cultures were repeated at least 3 times.

Conditioned medium. Conditioned medium from astrocyte cultures was collected after maintaining previously frozen astrocytes in astrocyte medium for 1 week. Cells were then washed with PBS, and the medium was changed to M41 medium. After 1 week, this medium was collected and stored at $-20^{\circ} \mathrm{C}$, and cells were again incubated for 1 week with M41 medium, which was also collected. Before being used in experiments, the conditioned medium was filtered through a $0.2-\mu \mathrm{m}$ filter and diluted 1:1 with fresh M41 medium to prevent exhaustion of essential factors. For the conditioned-medium experiments, the medium was refreshed every day.

For hyaluronan experiments, conditioned medium was treated with $50 \mathrm{U} / \mathrm{ml}$ hyaluronidase from Streptomyces hyalurolyticus (SigmaAldrich) overnight at $37^{\circ} \mathrm{C}$ prior to use. ACM treated with hyaluronidase diluent (20 mM sodium phosphate, $0.45 \% \mathrm{NaCl}, 0.01 \% \mathrm{BSA}$ ) alone overnight at $37^{\circ} \mathrm{C}$ served as a control. The following day, the medium was diluted 1:1 with fresh M41 medium and used for experiments.

Histochemistry and IHC. Mice were anesthetized and perfused with $4 \%$ PFA. Brains were post-fixed for 24 to 48 hours, removed, and cut along the midline. One half was embedded in paraffin, and the other half was cryoprotected in $30 \%$ sucrose, snap-frozen in OCT (Sakura Finetek Europe BV), and conserved at $-80^{\circ} \mathrm{C}$.

Paraffin-embedded tissue sections (6- $\mu$ m-thick) were deparaffinized and stained with $\mathrm{H} \& \mathrm{E}$. After heat-induced antigen retrieval in $0.01 \mathrm{M}$ citrate buffer ( $\mathrm{pH}$ 6), immunohistochemical staining was performed with Abs against GFAP (G3893, 1:1,000; Sigma-Aldrich); the isoform delta of GFAP (GFAP $\delta$; 1:250; gift of E. Hol, University Medical Center Utrecht, Utrecht, Netherlands; ref. 36); HAS2 (H-60; 1:50; Santa Cruz Biotechnology Inc.); GLT1 (AB1783, 1:1,000; EMD Millipore); MBP (MAB387; 1:50; EMD Millipore); PLP (MCA839G; 1:3,000; AbD Serotec); and $\beta$ APP (A8717, 1:750; Sigma-Aldrich). Immunopositivity was detected with DAB chromogen.

Frozen tissue sections (12- $\mu$ m-thick) were used for fluorescence IHC. Antigen retrieval was achieved as described above. SMI32 staining required blocking of endogenous peroxidase activity by incubation in methanol containing $3 \% \mathrm{H}_{2} \mathrm{O}_{2}$. Tissue sections were blocked for 1 hour in PBS, $5 \%$ normal goat serum, $0.1 \%$ BSA, and 0.3\% Triton X-100 and incubated with primary Abs overnight at $4^{\circ} \mathrm{C}$. For cell culture stains, no antigen retrieval was performed, and slides were immediately incubated in blocking solution for 1 hour and then in primary Ab overnight.
The Abs used targeted CD11b (M1/70.15.11.5.2; 1:50; Hybridoma Bank); GFAP; GFAPס; nestin (Rat-401-s; 1:100; Hybridoma Bank); Has2; Nk2 homeobox 2 (NKX2.2, 74.5A5-s; 1:50; Hybridoma Bank); nonphosphorylated neurofilaments (SMI32; 1:1,000; Covance); MBP (SMI99p; 1:2,000; Covance); MOG (MAB5680; 1:500; EMD Millipore); and olig2 (1:10,000; gift of J.H. Alberta, Harvard University, Boston, Massachusetts, USA). After incubating with secondary Abs (Alexa Fluor 488- or Alexa Fluor 594-tagged secondary Abs; 1:1,000; Molecular Probes), sections were counterstained with DAPI (10 ng/ml; Molecular Probes) and embedded in Fluoromount $\mathrm{G}$ (SouthernBiotech).

Human tissue was immunostained as described above for GFAP (1:1,000; EMD Millipore); GFAPס (1:250; gift of E. Hol, Netherlands Institute for Neuroscience, Amsterdam, Netherlands); $\operatorname{S100} \beta$ (1:200; Sigma-Aldrich); neurofilaments 70-200 kDa (1:10; Monosan); and vimentin (1:100; Dako).

Tissue sections and cell cultures were photographed using a Leica DM6000B microscope (Leica Microsystems). Omission of primary Abs yielded no significant staining. Pictures were acquired as TIFF images and optimized for brightness and contrast using Adobe Photoshop, version 7.0.

ISH. Antisense probes for Pdgfra and Plp were provided by D.H. Rowitch (UCSF, San Francisco, California, USA). Digoxigenin-labeled antisense RNA probes were made using plasmid DNA as a template and T7 as the RNA polymerase. RNA ISH was performed on frozen sections as previously described (60). Briefly, brain sections from mutant and WT mice were fixed with $4 \%$ PFA for 20 minutes, digested with Proteinase $\mathrm{K}$ $(10 \mu \mathrm{g} / \mathrm{ml})$ for 5 minutes, and post-fixed with $4 \%$ PFA for 15 minutes. After washing with PBS, sections were prehybridized in hybridization buffer for 2 hours at $65^{\circ} \mathrm{C}$ and incubated with the antisense probes overnight at $65^{\circ} \mathrm{C}$ in hybridization buffer. The following day, sections were washed with High Stringency wash (0.2× SSC plus 0.1\% Tween) for 1 hour at $65^{\circ} \mathrm{C}$ and with maleic acid-buffered solution $(100 \mathrm{mM}$ maleic acid, $150 \mathrm{mM} \mathrm{NaCl}, 2 \mathrm{mM}$ levamisole, and 0.1\% Tween) for 40 minutes at RT. After blocking with $2 \% \mathrm{BM}$ blocking reagent (Roche) and $20 \%$ sheep serum in maleic acid-buffered solution for 1 hour, the slides were incubated with anti-digoxygenin $\mathrm{Ab}$ (1:2,000; Roche) for 2 hours. Next, slides were washed with maleic acid-buffered solution for 3 hours, then with a solution containing $100 \mathrm{mM} \mathrm{NaCl}, 100 \mathrm{mM}$ Tris, $0.1 \%$ Tween, 2 $\mathrm{mM}$ levamisole for 20 minutes, followed by incubation with BM purple (Roche) overnight at RT. The next day, slides were washed in PBS, counterstained with $0.5 \%$ methyl green at $60^{\circ} \mathrm{C}$, dehydrated, and embedded with DePeX (Serva Electrophoresis).

Cell counts for IHC and ISH. For tissue sections, cells were counted in the corpus callosum. Total numbers of nestin-positive astrocytes and $P d g f r \alpha-, P l p-$, and Nkx2.2-positive oligodendrocytes were counted in at least 6 standardized fields per animal using a $\times 10$ objective lens. For cell cultures, the total number of GFAP-, olig2-, MBP-, and MOG-positive cells were counted in 10 randomly chosen fields within 1 well using a $\times 20$ objective lens. Immunopositive cells were expressed as a percentage of the total number of DAPI- or methyl green-positive nuclei. Plp-positive cells were expressed as absolute numbers per $100 \times 200 \mu \mathrm{m}$ area.

Electron microscopic analysis. Mice were perfused with saline buffer, followed by $2 \%$ glutaraldehyde and $4 \%$ PFA in $0.1 \mathrm{M}$ sodium cacodylate buffer ( $\mathrm{pH}$ 7.4). Brains were removed, and the corpus callosum was dissected, post-fixed in $1 \%$ osmium tetroxide and $1 \%$ potassium ferricyanide in $0.1 \mathrm{M}$ sodium cacodylate buffer, then dehydrated and embedded in epoxy resin. Longitudinally cut ultrathin sections 
were contrasted with uranyl acetate and lead citrate and viewed using a FEI Tecnai 12 electron microscope.

To evaluate myelination, we analyzed the thickness of the myelin sheaths on ultrathin sections of the corpus callosum from WT and mutant mice. The axonal diameters and $\mathrm{g}$ ratios (axon diameter/total fiber diameter) were determined in at least 400 axons per genotype using ImageJ software (NIH). When axons were not exactly circular, the shortest diameter was measured.

RNA isolation and PCR. RNA was extracted from frozen forebrain of WT and mutant mice or from cell culture wells with TRIzol according to the manufacturers' specifications. Subsequent reverse transcription to cDNA was performed with SuperScript III reverse transcriptase (Life Technologies). Quantitative real-time PCR was performed with a LightCycler 480 (Roche).

Transcript-specific primers were generated using Primer Express software (Applied Biosystems) and designed to overlap exon-exon boundaries to prevent genomic DNA amplification (Supplemental Table 5). The PCR reaction was performed on a 10- $\mu$ l volume containing SYBR Green PCR Mix (Roche), 1 pmol primers, and 150 ng cDNA. The relative abundance of transcript expression was calculated using the cycle threshold value and normalized to the endogenous controls $C y p b$, Rps14, Gapdh, and Akt. Each reaction was performed in duplicate.

Western blotting. Protein extracts were prepared from forebrain of WT and mutant animals in NP40 lysis buffer containing a protease inhibitor cocktail (Roche) with $1 \mathrm{mM}$ DTT. Supernatants (40 or $80 \mu \mathrm{g}$ total protein) were separated on $4 \%$ to $12 \%$ SDS-polyacrilamide precast gel (Invitrogen) and transferred onto PVDF membranes (Immobilon-P; EMD Millipore). The membranes were incubated with Abs against MBP, GFAP, GFAP $\delta$, and MOG and reprobed with $\beta$-actin (1:100,000; Sigma-Aldrich) to ensure equal loading. Proteins were visualized using alkaline phosphatase-coupled secondary Abs and ECF Western Blotting Detection Reagent (Amersham). The band intensity was quantified with ImageJ software.

Hyaluronan ELISA. Forebrain tissue was lysed in lysis buffer (50 mM Hepes, pH 7.5, 150 mM NaCl, 1 mM EDTA, 2.5 mM EGTA, $0.1 \%$ Triton X-100, $10 \%$ glycerol, and $1 \mathrm{mM} \mathrm{DTT}$ ) by grinding it 2-3 times with a Dounce tissue grinder (Sigma-Aldrich), followed by a 20-minute incubation on ice. Protein concentrations were measured with a Bradford assay, and brain lysates were diluted to a concentration of $300 \mu \mathrm{g} / \mathrm{ml}$ in lysis buffer. Immediately prior to performing the ELISA, lysates were further diluted to a concentration of $60 \mu \mathrm{g} / \mathrm{ml}$ in RD5-18 (R\&D Systems). Conditioned-medium samples were collected as described above and diluted 1:5 in RD5-18. Of these ACM and brain lysate dilutions, $50 \mu \mathrm{l}$ was used for a sandwich ELISA to determine hyaluronan levels according to the manufacturer's protocol (DHYALO; R\&D Systems). At the end of the protocol, plates were measured within 30 minutes on $450 \mathrm{~nm}$ with a wavelength correction at $540 \mathrm{~nm}$. A 4-parameter logistic standard curve was generated, and samples were fitted on the curve to acquire a concentration value. Further analyses were done with SPSS software as described in the Statistics section.

Statistics. For behavioral tests, PCR, IHC, Western blotting, ELISA, electron microscopy, and ISH, all data collected were used for analysis. For the culture experiments, data were excluded when cultures did not make it to the endpoint of the study (determined as described in the Astrocyte-OPC cocultures section) due to infection or when the batch of OPCs did not show MBP-positive cells in all conditions, which occasionally happened. No outliers were detected.
Detection of a genotype effect in behavioral tests was performed using ANOVA (genotype) or repeated-measures ANOVA (genotype $\times$ time). For the multiple parameters obtained in the home cage, stringent Bonferroni's correction was applied to correct for multiple tests (i.e., the significance level for ANOVA was set at $P<0.0025$ ).

The difference in the lifespan of WT and mutant mice was determined by a Kaplan-Meier log-rank analysis. All data were analyzed with SPSS Statistics 20 software (IBM SPSS Statistics). If the dataset met the assumptions of a parametric test and was not significant on the Shapiro-Wilk test for normality, an independent Student's $t$ test was used to analyze the data. Otherwise, a Mann-Whitney $U$ test was used. Paired-samples tests (paired-samples $t$ test or the nonparametric Wilcoxon signed-rank test) were used for coculture experiments, in which the OPCs from a single isolation were subjected to all the conditions tested in that specific experiment. For trend analysis, a 1-way ANOVA with polynomial contrasts was performed.

When multiple genotypes were tested, a Bonferroni's correction of the $\alpha$ was performed (standard $\alpha=0.05$ ). All data were analyzed using 2-sided tests. Pearson's correlation coefficient $r$ was used as the effect size, where an $r$ value greater than 0.50 was considered a large effect. For the percentages of small axons, a Pearson's $\chi^{2}$ test was used, with Cramer's V as the effect size $(\mathrm{V}>0.50$ was considered a large effect). See Supplemental Table 4 for all the tests used with descriptive statistics, $P$ values, and effect sizes. Data are expressed as the mean \pm SEM, unless otherwise noted.

Study approval. All procedures involving mice were performed in strict compliance with the animal welfare policies of the Dutch government and approved by the IACUC of the VU University of Amsterdam. Written informed consent was provided by patients or their parents for the collection and use of patients' samples, and the Medical Ethics Committee of the VU University Medical Center approved the procedures.

\section{Author contributions}

$\mathrm{SD}$ and $\mathrm{MB}$ performed experiments and analyses, wrote the manuscript, and contributed to the project design. MB and NLP performed stainings on paraffin material and Western blotting. EP and LW performed and TEMA supervised the quantitative real-time PCR experiments. SD, NL, STH, ALFvD, and AvdK performed the coculture studies. GJ performed mouse genotyping. VMH supervised genotyping, mouse breeding, and experiments. $\mathrm{MB}$ and $\mathrm{CV}$ analyzed the eye pathology. JK, MK, and JW performed EM experiments. ML performed the behavioral phenotyping. GCS and MSvdK designed the mutant mice. $\mathrm{VMH}$ designed the astrocyte-OPC coculture system. VMH and MSvdK designed and supervised the project, obtained funding, and wrote the manuscript.

\section{Acknowledgments}

We thank Anne-Marie van Dam (VU University, Amsterdam, Netherlands) for assistance with the ELISA; Elly M. Hol (University Medical Centre, Utrecht, Netherlands) for the GFAP $\delta$ Ab; and Arthur Bergen and Theo Gorgels (Netherlands Institute for Neuroscience, Amsterdam, Netherlands) for help with analysis of the eyes. This study was financially supported by the Dutch Organization for Scientific Research (NWO Spinoza grant 2008; ZonMw TOP grant 40-00812-98-11005); the Fonds NutsOhra 
(SNO-T-08-32); the Phelps Foundation (grant 2011.040); and the Optimix Foundation for Scientific Research. V.M. Heine is supported by ZonMw VIDI research grant 91712343 and by the Royal Dutch Academy of Arts and Sciences (KNAW) Van Leersumfonds.
Address correspondence to: Marjo S. van der Knaap, Department of Child Neurology, VU University Medical Centre, De Boelelaan 1117, 1081 HV Amsterdam, Netherlands. Phone: 31.0.20.4444856; E-mail:ms.vanderknaap@vumc.nl.
1. van der Knaap MS, Pronk JC, Scheper GC. Vanishing white matter disease. Lancet Neurol. 2006;5(5):413-423.

2. Leegwater PA, et al. Subunits of the translation initiation factor eIF2B are mutant in leukoencephalopathy with vanishing white matter. Nat Genet. 2001;29(4):383-388.

3. van der Knaap MS, et al. Mutations in each of the five subunits of translation initiation factor eIF2B can cause leukoencephalopathy with vanishing white matter. Ann Neurol. 2002;51(2):264-270.

4. Labauge $P$, et al. Natural history of adult-onset eIF2B-related disorders: a multi-centric survey of 16 cases. Brain. 2009;132(pt 8):2161-2169.

5. van der Lei HDW, et al. Genotype-phenotype correlation in vanishing white matter disease. Neurology. 2010;75(17):1555-1559.

6. Fogli A, et al. The effect of genotype on the natural history of eIF2B-related leukodystrophies. Neurology. 2004;62(9):1509-1517.

7. van der Knaap MS, et al. eIF2B-related disorders: antenatal onset and involvement of multiple organs. Am J Hum Genet. 2003;73(5):1199-1207.

8. Scheper GC, Proud CG, van der Knaap MS. Defective translation initiation causes vanishing of cerebral white matter. Trends Mol Med. 2006;12(4):159-166.

9. Liu R, et al. Severity of Vanishing White Matter disease does not correlate with deficits in eIF2B activity or the integrity of eIF2B complexes. Hum Mutat. 2011;32(9):1036-1045.

10. Li W, Wang XM, van der Knaap MS, Proud CG. Mutations linked to leukoencephalopathy with vanishing white matter impair the function of the eukaryotic initiation factor 2B complex in diverse ways. Mol Cell Biol. 2004;24(8):3295-3306.

11. Richardson JP, Mohammad SS, Pavitt GD. Mutations causing childhood ataxia with central nervous system hypomyelination reduce eukaryotic initiation factor $2 \mathrm{~B}$ complex formation and activity. Mol Cell Biol. 2004;24(6):2352-2363.

12. Horzinski L, et al. Eukaryotic initiation factor 2B (eIF2B) GEF activity as a diagnostic tool for EIF2B-related disorders. PLoS One. 2009;4(12):e8318.

13. van Kollenburg B, et al. egulation of protein synthesis in lymphoblasts from vanishing white matter patients. Neurobiol Dis. 2006;21(3):496-504.

14. Kantor $\mathrm{L}$, et al. Heightened stress response in primary fibroblasts expressing mutant IF $2 B$ genes from $\mathrm{CACH} / \mathrm{VWM}$ leukodystrophy patients. Hum Genet. 2005;118(1):99-106.

15. Fogli A, et al. Decreased guanine nucleotide exchange factor activity in eIF2B-mutated patients. Eur J Hum Genet. 2004;12(7):561-566.

16. Bugiani M, Boor I, Powers JM, Scheper GC, van der Knaap MS. Leukoencephalopathy with Vanishing White Matter: a review. J Neuropathol Exp Neurol. 2010;69(10):987-996.

17. Wong K, et al. Foamy cells with oligodendroglial phenotype in childhood ataxia with diffuse cen- tral nervous system hypomyelination syndrome. Acta Neuropathol. 2000;100(6):635-646.

18. Dietrich J, et al. EIF2B5 mutations compromise $\mathrm{GFAP}(+)$ astrocyte generation in vanishing white matter leukodystrophy. Nat Med. 2005;11(3):277-283

19. Rodriguez D, et al. Increased density of oligodendrocytes in childhood ataxia with diffuse central hypomyelination $(\mathrm{CACH})$ syndrome: neuropathological and biochemical study of two cases. Acta Neuropathol. 1999;97(5):469-480.

20. Bugiani $M$, et al. Defective glial maturation in Vanishing White Matter disease. JNeuropathol Exp Neurol. 2011;70(1):69-82.

21. Bugiani M, et al. Hyaluronan accumulation and arrested oligodendrocyte progenitor maturation in vanishing white matter disease. Brain. 2013;136(pt 1):209-222.

22. Back SA, et al. Hyaluronan accumulates in demyelinated lesions and inhibits oligodendrocyte progenitor maturation. Nat Med. 2005;11(9):966-972.

23. Fogli A, et al. Cree leukoencephalopathy and $\mathrm{CACH} / \mathrm{VWM}$ disease are allelic at the EIF2B5 locus. Ann Neurol. 2002;52(4):506-510.

24. Sofroniew MV. Molecular dissection of reactive astrogliosis and glial scar formation. Trends Neurosci. 2009;32(12):638-647.

25. Jennings MD, Zhou Y, Mohammad-Qureshi SS, Bennett D, Pavitt GD. eIF2B promotes eIF5 dissociation from eIF2 center dot GDP to facilitate guanine nucleotide exchange for translation initiation. Genes Dev. 2013;27(24):2696-2707.

26. Marom L, Ulitsky I, Cabilly Y, Shamir R, ElroyStein O. A point mutation in translation initiation factor eIF2B leads to function- and time-specific changes in brain gene expression. PLoS One. 2011;6(10):e26992.

27. van der Knaap MS, Kamphorst M, Barth PG, Kraaijeveld CL, Gut E, Valk J. Phenotypic variation in leukoencephalopathy with vanishing white matter. Neurology. 1998;51(2):540-547.

28. Hata Y, et al. An autopsy case of infantile-onset vanishing white matter disease related to an EIF2B2 mutation (V85E) in a hemizygous region. Int J Clin Exp Pathol. 2014;7(6):3355-3362.

29. Depienne C, et al. Brain white matter oedema due to $\mathrm{ClC}-2$ chloride channel deficiency: an observational analytical study. Lancet Neurol. 2013;12(7):659-668.

30. Geva M, et al. A mouse model for eukaryotic translation initiation factor $2 \mathrm{~B}$-leucodystrophy reveals abnormal development of brain white matter. Brain. 2010;133(pt 8):2448-2461.

31. Bjartmar C, Yin XH, Trapp BD. Axonal pathology in myelin disorders. J Neurocytol. 1999;28(4-5):383-395.

32. Robain O. The jimpy mouse. Acta Zool Pathol Antverp. 1977;68(68):68-92.

33. Rosenfeld J, Freidrich VL. Axonal swellings in jimpy mice - does lack of myelin cause neuronal abnormalities. Neuroscience. 1983;10(3):959-966.

34. Kamphuis W, et al. GFAP isoforms in adult mouse brain with a focus on neurogenic astrocytes and reactive astrogliosis in mouse models of Alzheimer disease. PLoS One. 2012;7(8):e42823.

35. Middeldorp J, Hol EM. GFAP in health and disease. Prog Neurobiol. 2011;93(3):421-443.

36. Mamber C, Kamphuis W, Haring NL, Peprah N, Middeldorp J, Hol EM. GFAP $\Delta$ expression in glia of the developmental and adolescent mouse brain. PLoS One. 2012;7(12):e52659.

37. Huyghe A, et al. Developmental splicing deregulation in leukodystrophies related to EIF2B mutations. PLoS One. 2012;7(6):e38264.

38. Xu HW, et al. Bergmann glia function in granule cell migration during cerebellum development. Mol Neurobiol. 2013;47(2):833-844.

39. Metea MR, Newman EA. Calcium signaling in specialized glial cells. Glia. 2006;54(7):650-655.

40. Eckstein AK, Reichenbach A, Jacobi P, Weber P, Gregor M, Zrenner E. Hepatic retinopathia. Changes in retinal function. Vision Res. 1997;37(12):1699-1706.

41. Hammond TR, et al. Astrocyte-derived endothelin-1 inhibits remyelination through notch activation. Neuron. 2014;81(3):588-602.

42. Wang YP, et al. Astrocytes from the contused spinal cord inhibit oligodendrocyte differentiation of adult oligodendrocyte precursor cells by increasing the expression of bone morphogenetic proteins. J Neurosci. 2011;31(16):6053-6058.

43. Arai K, Lo EH. Astrocytes protect oligodendrocyte precursor cells via MEK/ERK and PI3K/Akt signaling. J Neurosci Res. 2010;88(4):758-763.

44. Chernoff GF. Shiverer - an autosomal recessive mutant mouse with myelin deficiency. J Hered. 1981;72(2):128.

45. Lu JF, et al. A mouse model for X-linked adrenoleukodystrophy. Proc Natl Acad Sci US A. 1997;94(17):9366-9371.

46. Hess B, et al. Phenotype of arylsulfatase Adeficient mice: Relationship to human metachromatic leukodystrophy. Proc Natl Acad Sci U S A. 1996;93(25):14821-14826.

47. Behrendt R, Roers A. Mouse models for Aicardi-Goutieres syndrome provide clues to the molecular pathogenesis of systemic autoimmunity. Clin Exp Immunol. 2014;175(1):9-16.

48. Hagemann TL, Connor JX, Messing A. Alexander disease-associated glial fibrillary acidic protein mutations in mice induce rosenthal fiber formation and a white matter stress response. J Neurosci. 2006;26(43):11162-11173.

49. Verheijden S, et al. Peroxisomal multifunctional protein-2 deficiency causes neuroinflammation and degeneration of Purkinje cells independent of very long chain fatty acid accumulation. Neurobiol Dis. 2013;58:258-269.

50. Ramakrishnan H, et al. Increasing sulfatide synthesis in myelin-forming cells of arylsulfatase A-deficient mice causes demyelination and 


\section{RESEARCH ARTICLE}

neurological symptoms reminiscent of human metachromatic leukodystrophy. J Neurosci. 2007;27(35):9482-9490.

51. Messing A, Head MW, Galles K, Galbreath EJ, Goldman JE, Brenner M. Fatal encephalopathy with astrocyte inclusions in GFAP transgenic mice. Am J Pathol. 1998;152(2):391-398.

52. Lin $Y$, et al. Impaired eukaryotic translation initiation factor 2B activity specifically in oligodendrocytes reproduces the pathology of vanishing white matter disease in mice. J Neurosci. 2014;34(36):12182-12191.

53. Cabilly Y, et al. Poor cerebral inflammatory response in eIF2B knock-in mice: implications for the aetiology of vanishing white matter disease. Plos One. 2012;7(10):e46715.

54. Dever TE. Gene-specific regulation by general translation factors. Cell. 2002;108(4):545-556.

55. Turon-Vinas E, et al. Vanishing white matter disease in a spanish population. JCent Nerv Syst Dis. 2014;6:59-68.

56. Oberheim NA, Goldman SA, Nedergaard M. Heterogeneity of astrocytic form and function. Methods Mol Biol. 2012;814:23-45.

57. Matyash V, Kettenmann H. Heterogeneity in astrocyte morphology and physiology. Brain Res Rev. 2010;63(1-2):2-10.

58. Loos M, et al. Sheltering behavior and locomotor activity in 11 genetically diverse common inbred mouse strains using home-cage monitoring. PLoS One. 2014;9(9):e108563.

59. Sim FJ, McClain CR, Schanz SJ, Protack TL, Windrem MS, Goldman SA. CD140a identifies a population of highly myelinogenic, migration-competent and efficiently engrafting human oligodendrocyte progenitor cells. Nat Biotechnol. 2011;29(10):934-941.

60. Heine VM, Rowitch DH. Hedgehog signaling has a protective effect in glucocorticoid-induced mouse neonatal brain injury through an 11 beta HSD2-dependent mechanism. J Clin Invest. 2009;119(2):267-277. 